\section{The role of healthy nutrition and diet in the prevention of non-communicable diseases among the aged}

\section{Israel Ayenigbara}

Department of Human Kinetics and Health Education, University of Ibadan, Ibadan, Nigeria

\begin{abstract}
Non-communicable diseases (NCDs) kill approximately 41 million individuals every year, proportional to $71 \%$ of all deaths universally. This paper discuses the role of healthy nutrition and diet in the prevention of NCDs among the elderly people. This study is a theoretical research in which NCDs and the older people was discussed, and researched backed nutritional recommendations for the prevention of common NCDs and other geriatric illnesses was extensively discussed under; Increment in vegetable and fruit consumption, Lessening of saturated fat intake, Increment in dietary fiber consumption, Lessening of dietary sodium intake, Increment in dietary potassium consumption, and reduction in alcohol consumption. It was concluded that healthy nutrition helps in the prevention of NCDs and chronic diseases among the elderly people; therefore, healthy nutrition should be a preferred strategy tool in the prevention of NCDs and chronic diseases among the elderly population. It was however recommended that; keeping up good weight, increasing vegetable and natural fruits product consumption, reduction in the consumption of saturated fat, increasing dietary fiber consumption, decrease in dietary sodium and increment of dietary potassium intake, and reducing the rate of alcohol use; all helps in the prevention of NCDs and other illnesses affecting the elderly people.
\end{abstract}

\section{Introduction}

In the world today, the elderly population is experiencing a greater burden of chronic and NCDs than any time in recent memory as a result of increasing life expectancy and exposure to a lifetime of risk factors. ${ }^{1}$ Therefore, some nutritioninduced chronic conditions are of general health concerns, since they are of great significance in the causes of morbidity and mortality universally. Morbidity from chronic diseases could be characterized as a decrease in the functional ability of the body, movement restrictions, a lower level of physical activity, an inability to look after oneself, frailty, and a poorer quality of life. ${ }^{1}$

Non-communicable diseases (NCDs), otherwise called chronic diseases are usually of long duration and is the after effect of a combining multifaceted factors of hereditary, physiological, environmental and behavioral. ${ }^{2}$ It should be noted that, there are many types of NCDs which affects the health of elderly people, to name a few, the major ones include; cardiovascular diseases (heart attacks and stroke); different types of cancer; chronic respiratory diseases (such as chronic obstructive pulmonary disease and asthma); and diabetes. ${ }^{2,3}$ Statistically, NCDs disproportionately affects people in low and middle income countries, where in excess of seventy five percent $(75 \%)$ of worldwide NCDs deaths of 32 million occurs. ${ }^{2}$ Furthermore, NCDs kills approximately 41 million individuals every year, proportional to $71 \%$ of all deaths universally. ${ }^{2}$ In addition, every year, 15 million people died from a NCDs between the ages of 30 and 69 years; as more than $85 \%$ of these untimely deaths happen in low-and middle salary nations. ${ }^{2}$ Of all the various types of NCDs, cardiovascular diseases represents the most NCDs deaths; with a record of 17.9 million deaths every year, trailed by cancers $(9.0$ million), respiratory diseases (3.9 million), and diabetes (1.6 million). Shockingly, the four groups of diseases mentioned above, represents more than $80 \%$ of all untimely NCDs deaths worldwide. ${ }^{2}$

Although, people of all age groups, races, regions and countries are affected by NCDs, but these conditions are often associated with older population, ${ }^{2}$ as evidence affirms that 15 million of all deaths attributed to NCDs occurs between the ages of 30 and 69 years. Unfortunately, the death statistics from NCDs are frightening, as it is a challenge to health advocates on why such preventable diseases are causing high morbidity and mortality rate. It should be noted that NCDs are preventable diseases; hence, the need for a review on the role of healthy nutrition and diet in the prevention of NCDs, with the focus on the aged.

\section{Methods of research}

This is a theoretical research and the review focuses on the role of healthy nutrition and diet in the prevention of NCDs among the aged. Literature was recovered in Web of Science, Google scholar, PubMed and Springer Link databases. Furthermore, major international and national health
Correspondence: Israel Ayenigbara, Department of Human Kinetics and Health Education, University of Ibadan, Ibadan, Nigeria. Tel.: 08139177538.

E-mail: histrealite2647@gmail.com

Key words: Healthy nutrition; prevention; non-communicable diseases; aged.

Conflict of interest: the author declares no potential conflict of interests.

Dedication: the article is dedicated to all the elderly people around the world.

Received for publication: 1 December 2018. Revision received: 17 February 2019. Accepted for publication: 29 March 2019.

This work is licensed under a Creative Commons Attribution-NonCommercial 4.0 International License (CC BY-NC 4.0).

(C) Copyright I. Ayenigbara, 2019

Licensee PAGEPress, Italy

Geriatric Care 2019; 5:7961

doi:10.4081/gc.2019.7961

sites, to name a few, the World health Organization, U.S. Department of Health and Human Services and U.S. Department of Agriculture, and New Zealand Ministry of Health; were searched to fetch related information. Year of publication was not a factor as the researcher needed to get important data on the effect of nutrition in the prevention of NCDs among the aged. Careful screening was done to ensure that significant data was incorporated into this review. Subsequently, References were altogether looked at, and reference arrangements of every single study included in this review were deliberately inspected.

\section{Non-communicable diseases}

Non-communicable diseases (NCDs) are no longer only a problem for developed countries, as these former disease of affluence are now the leading causes of death in all regions, and mostly affects the young to old adults population. ${ }^{2}$ In New Zealand for instance, cancer and ischemic heart disease were the main sources of death from 1987 to $2005 .{ }^{1}$ Additionally, in 2005, malignancy (cancer) represented 29.4 percent of deaths among adults in New Zealand, while coronary heart diseases represented 21.4 percent of deaths. ${ }^{4}$ While in the UK for instance, in 2015 , coronary heart diseases were the most widely recognized causes of death for male adults, which was two times more than the 
second causes of death in this gender; dementia and Alzheimer's diseases are second and third respectively. ${ }^{5}$ For female adults, dementia and Alzheimer's was the most widely recognized causes of death, trailed by coronary heart diseases, ${ }^{5}$ for both male and female adults, dementia and Alzheimer's disease were the main causes of death. ${ }^{5}$ Significantly, diseases in general represented $30.0 \%$ of deaths in male and $24.8 \%$ of deaths in females in 2015 in the UK. ${ }^{5}$ In addition to the morbidity and mortality related with chronic diseases in adults, other health conditions such as, reduced nutrient ingestion, reduced transportation of nutrients in the body, and reduced digestion will occur. ${ }^{1}$ Furthermore, People experiencing one or more chronic diseases will experience a reduced appetite, difficulty with activities of daily living and mobility, dementia and depression, and such impacts might be exacerbated by drugs used to control these chronic diseases. ${ }^{6}$

Chronic diseases in elderly people mirrors the amassing of exposures to risk factors for the duration of one life's course, unfortunately, it is in the old age that most chronic diseases will become visible and apparent. ${ }^{7}$ Understandably, the burden from some of these nutrition related chronic diseases is large, but the potential advantages from moderate enhancements in nutrition and physical activities in preventing them are additionally impressive. ${ }^{8}$ Unmistakably, there are advantages for elderly population in lessening their danger of chronic diseases in the future through the selection of health promoting practices which are, adequate rest and sleep, and most importantly, healthy eating, adequate exercise and physical activity. Furthermore, the Intervention in old age to prevent some nutrition-related chronic diseases could be achieved and realistic through nutrition modifications, such as eating the right food. 7,9

\section{Nutrition recommendations for the prevention of non-communicable diseases in the aged}

Healthy diet is important for maintaining good health and preventing the risk of NCDs. ${ }^{10,11}$ In addition, healthy eating is characterized as, eating different forms of nutritious foods, eating balanced diet and following the standard food and nutrition guidelines. ${ }^{1}$ Importantly, in the prevention of NCDs among the aged, the following scientifically researched based recommendations are indispensable. ${ }^{1}$

\section{Increment in vegetable and fruit consumption}

Eating of vegetable and adequate fruit consumption are both protective against cardiovascular diseases and cancers. ${ }^{8}$ In support of the aforementioned, a research on the Association between the Consumption of Fruits and Vegetables, and Risk of Colorectal Adenoma revealed that, consumption of fruits specifically prevents colorectal cancer. ${ }^{12}$ In addition, the intake of vegetables and fruits significantly reduces the risk of bladder cancer. ${ }^{13}$ Furthermore, the World Cancer Report stated that vegetables and natural fruits are a likely defensive factor against cancers of the mouth, larynx, pharynx, throat and stomach, and natural fruits prevent against the cancer of the lung as well. ${ }^{14}$ Also, from a critical review on the associations between the intake of vegetables and fruits, and the risk of several chronic diseases, the study revealed that a high daily intake of vegetables and fruits promote health, and the promotion of vegetable and fruit consumption by nutrition and health policies is a preferable strategy to reduce the burden of several chronic diseases. ${ }^{15}$ Furthermore, high consumption of Fruits and vegetables reduces dementia and risk of cognitive impairment in elderly people. ${ }^{16}$ Also, increasing fruit and vegetable consumption helps to prevent the risk of NCDs. ${ }^{17}$ In addition, including fruits and vegetables as part of the daily diet reduces the risk of some NCDs, including cardiovascular diseases and certain types of cancer. ${ }^{17}$ Although, limited evidence suggests that when consumed as part of a healthy diet low in fat, sugars and salt/sodium, fruits and vegetables helps to prevent weight gain and reduce the risk of obesity which is an independent risk-factor for NCDs. ${ }^{17}$ As part of a healthy diet low in fat, sugars and sodium, the World Health Organization (WHO) recommends consuming more than 400 grams of fruits and vegetables per day helps to improve the overall health and reduces the risk of some NCDs. ${ }^{17}$ Important Sources of fruits and vegetablesrich foods includes; watermelon, strawberries, cantaloupe, peaches, oranges, skim milk, cucumber, lettuce, zucchini, celery, plain yourgut, tomatoes, bell pepper, cauliflower, cabbage, grapefruit, coconut water, cottage cheese, apple, bananas, raisins, pineapple, and so on..$^{18,19}$

\section{Reduce saturated fat consumption}

Reduction in the saturated fat consumption is very important to the health of an elderly individual, and also for the prevention of some NCDs. ${ }^{11}$ For example, consumption of saturated fats causes bone fractures and breakage; this is most common among elderly people. ${ }^{20}$ It is important that Saturated fats consumption should be substituted with unsaturated fats, as they pose fewer disadvantages to health than saturated fats. ${ }^{21}$ In line with the aforementioned, a scientific research on the Substitution of dietary polyunsaturated fatty acids for saturated fatty acids to know if it will decrease the LDL apolipoprotein B-100 production rate in men with dyslipidemia (abnormal elevation of cholesterol in the blood), findings revealed that the substitution of dietary polyunsaturated fatty acids for saturated fatty acids decreases the production and number of LDL particles in men with dyslipidemia. ${ }^{22}$ Also, in furtherance of the early statement, a research into the dietary quality and patterns of NCDs risk of an Indian community in KwaZuluNatal, South Africa, findings from the study revealed that a reduced intake of fruit and vegetable, fibre, and saturated fat intake in the diet is highlighted and associated with higher number of NCDs cases. ${ }^{23}$ Notably, reduction and substitution of saturated fats with the polyunsaturated fats are still the standard dietary recommendation to prevent Coronary Heart Diseases and Cardiovascular Diseases. ${ }^{11,21}$ Hence, the notable sources of important fat the body needs are olive oil, nuts, seafood, vegetable oil, coconut oil, soybean oil, palm, palm kernel oil, coconut oil, canola, corn, olive, cotton seed, sunflower and peanut oil. ${ }^{19}$

\section{Increment in dietary fiber consumption}

Consumption of dietary fiber is very beneficial to the health of an elderly individual, and also helps in the prevention of some diseases..$^{11}$ According to a study on the latest systematic reviews and meta-analyses of Carbohydrate quality and human health, result from the Observational data suggest a $15-30 \%$ decrease in all-cause and cardiovascular related mortality, and incidence of coronary heart disease, stroke incidence and mortality, type 2 diabetes, and colorectal cancer when comparing the highest dietary fibre consumers with the lowest consumers, ${ }^{24}$ also, clinical trials show significantly lower bodyweight, systolic blood pressure, and total cholesterol when comparing higher with lower intakes of dietary fibre. ${ }^{24}$ Also from the study, dose- response curves suggested that higher intakes of dietary fibre could confer even greater benefit to protect against cardiovascular diseases, type 2 diabetes, and colorectal and breast cancer. ${ }^{24}$ In addition, an umbrella review of systematic reviews and meta-analyses of Dietary fiber consumption and health outcomes revealed that dietary recommendations that promote higher fiber intake as part of a healthy diet is good for overall health, also, Dietary fibre intake helps in the prevention of coronary artery 
disease, pancreatic cancer, and gastric cancer. ${ }^{25}$ Diverticulitis can be prevented by the consumption of dietary fiber. ${ }^{26}$ Also, consumption of vegetable fiber helps in the prevention of hypertension; this was affirmed by a study on the Association between Dietary Fiber Intake and Hypertension among U.S. Adults, findings revealed that intakes of total cereal, and vegetable fiber, but not fruit fiber, were associated with a decreased risk of hypertension in U.S. adults. $^{27}$ Furthermore, consumption of dietary fiber helps in preventing cancers, specifically ovarian cancer. ${ }^{28}$ In addition, a meta-analysis study on the effect of dietary fiber intake on the risk for colorectal adenoma, findings suggested that high dietary fiber intake is associated with reduced risk of colorectal adenoma. ${ }^{29}$ Also, there is some evidence for a protective effect of dietary fiber against oesophageal cancer. ${ }^{14}$ In addition, another meta-analysis study conducted on the effect of dietary fiber intake on inflammatory bowel disease, results indicated that the intake of dietary fiber was significantly associated with a decreased risk of inflammatory bowel disease. ${ }^{30}$ Notable sources of dietary fiber giving foods are; brown rice, oatmeal, popcorn, quinoa, breads, cereals, crackers, kernel, vegetables, fruits, and pasta. ${ }^{19}$

\section{Lessening the intake of dietary sodium}

Sodium chloride (salt) is the major sources of sodium in the eating regimen. ${ }^{31}$ Sodium is additionally present in diet as sodium bicarbonate and food additives, including monosodium glutamate, sodium phosphate, sodium carbonate and sodium benzoate. ${ }^{1}$ Although, sodium is an essential nutrient needed in the body, but much intake of sodium has a deleterious consequences on health. For example, an updated systematic review of salt and health outcomes confirmed that high sodium intake increases blood pressure and risk of kidney disease. ${ }^{32}$ Also, a research on large artery stiffness and its association with salt intake in young healthy black adults, findings revealed that, excessive salt intake is positively associated with early vascular ageing of large artery stiffness-independent of blood pressure-in young adults, especially in black individuals. ${ }^{33}$ Furthermore, higher salt intake increases the secretion of Cardiotrophin-1 (CT-1 serum in the body. ${ }^{34}$ Notably, Cardiotrophin-1 (CT-1) is a stressinduced cytokine that belongs to the interleukin 6 family, which is usually released by cells in response to potentially harmful stresses and plays a pivotal role in congestive heart failure, hypertension and arterial stiffness; research revealed that variations in dietary salt intake affect the serum CT-1 levels in Chinese adults. ${ }^{34}$ A systematic review and meta-analysis on the effect of lower sodium intake on health, findings were impressive; firstly, reduced sodium intake reduces blood pressure and has no adverse effect on blood lipids, catecholamine levels, or renal function. ${ }^{35}$ Secondly, moderate quality evidence in children shows that a reduction in sodium intake reduces blood pressure; lower sodium intake is also associated with a reduced risk of stroke and fatal coronary heart disease in adults, in summary, most people will benefit from reducing sodium intake.

\section{Increment in dietary potassium intake}

Consumption of higher intake of dietary potassium is advised instead of dietary sodium, as Potassium limits the impact of sodium chloride on blood pressure, thus helping to regulate blood pressure. ${ }^{1,8}$ Also, a comprehensive meta-analysis and systematic review on the effects of increased potassium intake on cardiovascular risk factors and disease, findings revealed that, increased potassium intake reduces blood pressure in people with hypertension, and has no adverse effect on blood lipid concentrations, catecholamine concentrations, or renal function in adults. ${ }^{36}$ Furthermore, higher potassium intake was associated with a $24 \%$ lower risk of stroke, these results further suggests that increased potassium intake is potentially beneficial to most people without impaired renal handling of potassium for the prevention and control of elevated blood pressure and stroke. ${ }^{36}$ In addition, the Intake of potassium with other micronutrients such as vitamin A, carotenoids, the vitamin B complex, vitamins $\mathrm{C}, \mathrm{D}$, and E, and coenzyme Q10 calcium, magnesium, iron, zinc, potassium, copper, manganese, helps in the prevention and therapy of periodontal disease ${ }^{37}$ Notably, vegetables and natural fruit products are decent sources of potassium, which adds to the connection between diets high in vegetables and fruits, and reduced risk of cardiovascular diseases. ${ }^{8}$ Other sources of potassium giving foods includes, winter squash, sweet potatoes, white beans, fat free yogurt, halibut, broccoli, cantaloupe, banana, low fat milk, salmon, raisins, and so on. ${ }^{19}$ Importantly, for adults with renal diseases, consumption of potassium should be reduced or avoided, as potassium does not help in the treatment and management of renal diseases. ${ }^{38}$

\section{Stop or reduce alcohol consumption and tobacco smoking}

There is convincing proof that alcohol of all types is the causes for various cancers. ${ }^{14}$ Notably, alcohol is related with cancers of the upper digestive tract, including the mouth, pharynx and larynx, and oesophagus. Also, a study on the associations of a healthy lifestyle with all stages of colorectal carcinogenesis, results revealed that healthy lifestyle; which includes reduced alcohol consumption is strongly associated with lower risk of all stages of colorectal neoplasm. ${ }^{39}$ Furthermore, alcohol consumption is a causative factor for hepatocellular carcinoma (HCC), which is the most common primary cancer of the liver responsible for an increasing number of cancer-related deaths, especially in developing economies of Asia and Africa. ${ }^{40}$ Also, alcohol abuse is a major risk factor for the management of elderly patients with oral cavity squamous cell carcinomas. ${ }^{41}$ Heavy alcohol consumption increases the risk of active tuberculosis in adults, ${ }^{42}$ as a cohort study on Taiwanese adults affirmed that heavy and regular alcohol consumption is associated with higher risks of active tuberculosis. ${ }^{42}$ Furthermore, alcohol consumption is associated with a higher likelihood of having probable rapid eye movement and sleep behavior disorder. ${ }^{43}$ Also, in a systematic review of the next-day effects of heavy alcohol consumption on cognitive performance, findings revealed that alcohol hangovers may involve impaired cognitive functions and performance of everyday tasks such as driving. ${ }^{44}$ In addition, alcohol consumption increases the susceptibility of a drinker to pneumococcal pneumonia (A bacterial infection caused by streptococcus pneumonia), and also worsens the severity of bacterial pneumonia in persons living with HIV.45

Alcohol consumption combined with cigarette smoking is also deleterious to health as, a study on the relationship of cigarette smoking and alcohol drinking in midlife with risk of cognitive impairment in late life concluded that smoking and regular alcohol drinking at midlife had a much stronger impact on the risk of cognitive impairment in late life. ${ }^{46}$ Furthermore, smoking and drinking is associated with higher prevalence of metabolic syndrome (a cluster of conditions that increase the risk of heart disease, stroke and diabetes). ${ }^{47}$ No level of alcohol consumption is good for the body, findings from a study on daily drinking and its association with increased mortality revealed that, minimum risk of low-level drinking frequency for all-cause mortality appears to be approximately three times; also, daily drinking, even at low levels, is detrimental to health. ${ }^{48}$

\section{Conclusions}

Nutrition is established as major modifiable determinants of NCDs. In the preven- 
tion of NCDs among the elderly people, nutritional modification is essential. This critical review on the association of healthy nutrition and diet in the prevention of NCDs among the aged revealed that, healthy nutrition helps in the prevention of NCDs or chronic diseases among this group. Therefore, from a scientific point of view, it was concluded that, healthy nutrition is justified, and should be a preferred strategy tool in the prevention of NCDs and chronic diseases among the elderly people.

\section{References}

1. Ministry of Health; 2013. Available from: https://www.health.govt.nz/publication/food-and-nutrition-guidelineshealthy-older-people-background-paper.

2. World Health Organization; 2018. Available from: http:/www.who.int/ news-room/fact-sheets/detail/noncommunicable-diseases

3. Cronin KA, Lake AJ, Scott S, et al. Annual Report to the Nation on the Status of Cancer, part I: National cancer statistics. Cancer 2018;124:2785-800.

4. Ministry of Health; 2012. Available from: https://www.health.govt.nz/publication/mortality-and-demographicdata-2009

5. Public Health England; 2017. Available from: https://www.gov.uk/government/ publications/health-profile-for-england/ chapter-2-major-causes-of-death-andhow-they-have-changed

6. Eggersdorfer M, Akobundu U, Bailey RL, et al. Hidden Hunger: solution for America's aging populations. Nutrients 2018;10:pii: E1210.

7. World Health Organization \& Food and Agricultural Organization; 2003. Available from: http://www.who.int/dietphysicalactivity/publications/trs916/dow nload/en/

8. Ministry of Health \& University of Auckland; 2003. Available from: http:// www.moh.govt.nz/notebook/nbbooks.ns f/0/A8D85BC5BAD17610CC256D970 072A0AA/\$file/nutritionandtheburdenofdisease.pdf

9. Ruxton $\mathrm{CH}$, Derbyshire E, ToribioMateas M. Role of fatty acids and micronutrients in healthy ageing: a systematic review of randomised controlled trials set in the context of European dietary surveys of older adults. J Hum Nutr Diet 2015;29:308-24.

10. Paglia L. WHO: healthy diet to prevent chronic diseases and caries. Eur J Paediatr Dent 2018;19:5.

11. World health Organization; 2018.
Available from: https://www.who.int/ dietphysicalactivity/publications/trs916/ summary/en/

12. Ben Q, Zhong J, Liu J, et al. Association between Consumption of Fruits and Vegetables and Risk of Colorectal Adenoma: A PRISMA-Compliant MetaAnalysis of Observational Studies. Medicine (Baltimore) 2015;94:e1599.

13. Liu H, Wang XC, Hu GH, et al. Fruit and vegetable consumption and risk of bladder cancer: an updated meta-analysis of observational studies. Eur J Cancer Prev 2015;24:508-16.

14. World Cancer Research Fund and American Institute for Cancer Research; 2018. Available from: https://www.wcrf. org/dietandcancer/resources-and-toolkit

15. Boeing $\mathrm{H}$, Bechthold A, Bub A, et al. Critical review: vegetables and fruit in the prevention of chronic diseases. Eur J Nutr 2012;51:637-63.

16. Jiang $X$, Huang J, Song D, et al. Increased consumption of fruit and vegetables is related to a reduced risk of cognitive impairment and dementia: meta-analysis. Front Aging Neurosci 2017;7:9:18.

17. World Health Organization; 2018. Available from: https://www.who.int/ elena/titles/fruit_vegetables_ncds/en/

18. Elliott B. 19 Water-rich foods that help you stay hydrated; 2017. Available from: https://www.healthline.com/nutrition/19 -hydrating-foods

19. U.S. Department of Health and Human Services and U.S. Department of Agriculture; 2015. Available from: http://health.gov/dietaryguidelines/201 5/guidelines

20. Mozaffari H, Djafarian K, Mofrad MD, et al. Dietary fat, saturated fatty acid, and monounsaturated fatty acid intakes and risk of bone fracture: a systematic review and meta-analysis of observational studies. Osteoporos Int 2018;29:1949-61.

21. Fattore E, Massa E. Dietary fats and cardiovascular health: a summary of the scientific evidence and current debate. Int J Food SciNutr 2018;69:916-27.

22. Drouin-Chartier JP, Tremblay AJ, Lépine MC, et al. Substitution of dietary $\omega-6$ polyunsaturated fatty acids for saturated fatty acids decreases LDL apolipoprotein B-100 production rate in men with dyslipidemia associated with insulin resistance: a randomized controlled trial. Am J ClinNutr 2018;107:26-34.

23. Naicker A, Venter CS, MacIntyre UE, et al. Dietary quality and patterns and noncommunicable disease risk of an Indian community in KwaZulu-Natal, South Africa. J Health Popul Nutr 2015;8; $33: 12$.
24. Reynolds A, Mann J, Cummings J, et al. Carbohydrate quality and human health: a series of systematic reviews and metaanalyses. Lancet 2019;393:434-45.

25. Veronese N, Solmi M, Caruso MG, et al. Dietary fiber and health outcomes: an umbrella review of systematic reviews and meta-analyses. Am J Clin Nutr 2018;107:436-44.

26. Špičák J, Kučera M, Suchánková G. Diverticular disease: diagnosis and treatment. VnitrLek 2018;64:621-34.

27. Sun B, Shi X, Wang T, et al. Exploration of the association between dietary fiber intake and hypertension among u.s. adults using 2017 American College of Cardiology/American Heart Association Blood Pressure Guidelines: NHANES 2007-2014. Nutrients 2018;10.pii: E1091.

28. Xu H, Ding Y, Xin X, et al. Dietary fiber intake is associated with a reduced risk of ovarian cancer: a dose- response meta-analysis. Nutr Res 2018;57:1-11.

29. Ben Q, Sun Y, Chai R, et al. Dietary fiber intake reduces risk for colorectal adenoma: a meta-analysis. Gastroenterology 2014;146:689-99.e6.

30. Liu X, Wu Y, Li F, et al. Dietary fiber intake reduces risk of inflammatory bowel disease: result from a metaanalysis. Nutr Res 2015;35:753-8.

31. Ix JH, Anderson CAM. Measurements of 24-hour urinary sodium and potassium excretion: importance and implications. JAMA 2018;319:1201-2.

32. Malta D, Petersen KS, Johnson C, et al. High sodium intake increases blood pressure and risk of kidney disease. From the Science of Salt: A regularly updated systematic review of salt and health outcomes (August 2016 to March 2017). J Clin Hypertens 2018 [Epub ahead of print].

33. Strauss M, Smith W, Kruger R, et al. Large artery stiffness is associated with salt intake in young healthy black but not white adults: the African-PREDICT study. Eur J Nutr 2018;57:2649-56.

34. Wang K, Chu C, Hu J, et al. Effect of salt intake on the serum cardiotrophin-1 levels in chinese adults. Ann Nutr Metab 2018;73:302-9.

35. Aburto NJ, Ziolkovska A, Hooper L, et al. Effect of lower sodium intake on health: systematic review and metaanalyses. BMJ 2013;3;346:f1326.

36. Aburto NJ, Hanson S, Gutierrez H, et al. Effect of increased potassium intake on cardiovascular risk factors and disease: systematic review and metaanalyses. BMJ 2013;346:f1378.

37. Dommisch H, Kuzmanova D, Jönsson $\mathrm{D}$, et al. Effect of micronutrient malnutri- 
tion on periodontal disease and periodontal therapy. Periodontol 2018;78: 129-53.

38. Hershey K. Renal diet. Nurs Clin North Am 2018; 53(4):481-489. doi: 10.1016/j.cnur.2018.05.005.

39. Erben V, Carr PR, Holleczek B, et al. Strong associations of a healthy lifestyle with all stages of colorectal carcinogenesis: results from a large cohort of participants of screening colonoscopy. Int $\mathrm{J}$ Cancer 2018 [Epub ahead of print].

40. Rawla P, Sunkara T, Muralidharan P, et al. Update in global trends and aetiology of hepatocellular carcinoma. Contemp Oncol (Pozn) 2018;22:141-50.

41. Gambotti L, Schwob E, Schouman T, et al. Are elderly patients presenting with squamous cell carcinoma of the oral cavity given the appropriate treatment? Surg Oncol 2018;27:715-21.

42. Yen YF, Hu HY, Lee YL, et al. Heavy alcohol consumption increases the risk of active tuberculosis in Taiwanese adults: a nation-wide population-based cohort study. Addiction 2017;112:2124-31.

43. Ma C, Pavlova M, Li J, et al. Alcohol consumption and probable rapid eye movement sleep behavior disorder. Ann Clin Transl Neurol 2018;5:1176-83.

44. Gunn C, Mackus M, Griffin C, et al. A systematic review of the next-day effects of heavy alcohol consumption on cognitive performance. Addiction 2018;113:2182-93.

45. Samuelson DR, Siggins RW, Ruan S, et al. Alcohol consumption increase susceptibility to pneumococcal pneumonia in a humanized murine HIV model mediated by intestinal dysbiosis. Alcohol 2018;pii:S0741-8329(18)30166-6.

46. Wu J, Dong W, Pan XF, et al. Relation of cigarette smoking and alcohol drinking in midlife with risk of cognitive impairment in late life: the Singapore Chinese Health Study. Age Ageing 2018 [Epub ahead of print].

47. Yu M, Xu CX, Zhu HH, et al. Associations of cigarette smoking and alcohol consumption with metabolic syndrome in a male Chinese population: a cross-sectional study. J Epidemiol 2014;24:361-9.

48. Hartz SM, Oehlert M, Horton AC, et al. Daily drinking is associated with increased mortality. Alcohol Clin Exp Res 2018;42:2246-55. 